REPRESENTATION THEORY

An Electronic Journal of the American Mathematical Society

Volume 10, Pages 299-313 (August 7, 2006)

S $1088-4165(06) 00267-6$

\title{
DIRAC OPERATORS AND LIE ALGEBRA COHOMOLOGY
}

\author{
JING-SONG HUANG, PAVLE PANDŽIĆ, AND DAVID RENARD
}

\begin{abstract}
Dirac cohomology is a new tool used to study unitary and admissible representations of semisimple Lie groups. It was introduced by Vogan and further studied by Kostant and ourselves. The aim of this paper is to study the Dirac cohomology of unitary modules for the Kostant cubic Dirac operator and its relation to nilpotent Lie algebra cohomology. We show that the Dirac cohomology coincides with the corresponding nilpotent Lie algebra cohomology in some cases. Along the way we prove some properties of Dirac cohomology that make it more accessible for calculation.
\end{abstract}

\section{INTRODUCTION}

In his lecture V3], D. Vogan presented an idea that one of the most important invariants of $(\mathfrak{g}, K)$-modules, $\mathfrak{u}$-homology related to a parabolic subalgebra $\mathfrak{q}=$ $\mathfrak{l} \oplus \mathfrak{u}$, could be related to the concept of Dirac cohomology introduced in V2 and studied further in [HP1] and [K4. The corresponding Dirac operator should be the Kostant's cubic Dirac operator corresponding to l.

Indeed, the two concepts agree up to an appropriate modular twist for finite dimensional modules. (They also agree with the $\mathfrak{u}^{-}$- cohomology corresponding to the opposite parabolic subalgebra.) This follows easily from a basic paper of Kostant [K1] which gives a Hodge decomposition of $\mathfrak{u}$-homology (or $\mathfrak{u}^{-}$-cohomology) using a certain Laplacean operator. Combined with a remark in K3 that this Laplacean is essentially the square of the Dirac operator, and with the easily proved fact that the Dirac cohomology equals $\operatorname{Ker} D=\operatorname{Ker} D^{2}$ in the finite-dimensional case, this gives the result.

The question raised by Vogan is whether some analogue of this remains true for (infinite-dimensional) unitary $(\mathfrak{g}, K)$-modules. This question is the main topic of the present paper. What we manage to do is to obtain a Hodge decomposition for

Received by the editors January 11, 2005 and, in revised form, March 6, 2006.

2000 Mathematics Subject Classification. Primary 22E47.

Key words and phrases. Semisimple Lie group, unitary representation, admissible representation, Dirac operator, Lie algebra cohomology.

The research of the first named author was partially supported by RGC-CERG grants of Hong Kong SAR and the National Nature Science Foundation of China.

The research of the second named author was partially supported by a grant from the Ministry of Science and Technology of the Republic of Croatia.

The second and third named authors were also supported by the joint French-Croatian program COGITO

Parts of this work were done during the authors' visits to CNRS, University of Paris VII, Mathematisches Forschungsinstitut Oberwolfach, and the Institute of Mathematical Sciences and the Department of Mathematics at the National University of Singapore. The authors thank these institutions for their generous support and hospitality.

(C)2006 American Mathematical Society Reverts to public domain 28 years from publication 
$\mathfrak{u}$-homology (and $\mathfrak{u}^{-}$-cohomology) of unitary modules in certain special cases: the pair $(\mathfrak{g}, \mathfrak{k})$ must be Hermitian symmetric, the parabolic subalgebra $\mathfrak{q}=\mathfrak{l} \oplus \mathfrak{u}$ must be $\theta$-stable, $\mathfrak{l}$ must be contained in $\mathfrak{k}$ and $\mathfrak{u}$ must contain $\mathfrak{p}^{+}$. (Here $\mathfrak{g}=\mathfrak{k} \oplus \mathfrak{p}$ is the Cartan decomposition corresponding to a Cartan involution $\theta$.) Under these assumptions we obtain results completely analogous to the finite-dimensional case (see Theorem 7.11, which is the main result of this paper). Let us also note that there are examples when these results do not hold, as well as examples when the results hold in a more general setting than the one described above.

Along the way, we obtain some properties of Dirac cohomology. Most notably, in certain cases it can be calculated in stages. This is analogous, but much simpler than the Hochschild-Serre spectral sequence for $\mathfrak{u}$-homology or $\mathfrak{u}^{-}$-cohomology (see for example [V1]). A possible further advantage of Dirac cohomology over $\mathfrak{u}$-homology is the fact (noted in [V3]) that Dirac cohomology is "more invariant" in the sense that it depends only on $\mathfrak{l}$ and not on $\mathfrak{u}$, while several parabolics may have the same $\mathfrak{l}$.

All this is also related to the fact that in cases when they agree, the Dirac cohomology is in fact a coarser invariant than the corresponding $\mathfrak{u}$-homology. Namely, the grading is almost completely lost, and only the even and odd parts make sense. Related to this is the fact that in some cases there is a six-term exact sequence of Dirac cohomology in place of the usual long exact sequences of $\mathfrak{u}$-homology and $\mathfrak{u}^{-}$-cohomology. Thus, Dirac cohomology seems to have more of a K-theoretic than a cohomological flavor.

Acknowledgments. Among the many people who expressed interest in our work and gave us useful comments and suggestions, we would especially like to thank A. Alekseev, M. Duflo, T. Kobayashi, S. Kumar, E. Meinrenken, K. Nishiyama, E.-C. Tan, T. Uzawa, M. Vergne, D. Vogan, N. Wallach and H. Yamashita.

For readers who need a longer introduction to the subject, one possible source is $\mathrm{HP}$ 2. For those interested in further topics related to the subject of this paper (and of [HP1]), we suggest $\mathrm{AM}$ ] and $[\mathrm{Ku}$.

Finally, let us mention that there is a longer version of this paper posted on arXiv:math.RT. The present, shorter version was (rightfully) suggested by the referee, who noted that there were too many side results in the original version which were not really necessary to obtain the main result. We would like to thank the referee for his (or her) useful suggestions.

\section{Decomposing Kostant's Cubic Dirac operator}

In [K2], B. Kostant introduces cubic Dirac operators, which are a generalization of Dirac operators first used by Parthasarathy $[\mathrm{P}]$ in representation theory. The setting is the following. Let $\mathfrak{g}$ be a complex semisimple Lie algebra, and denote by $B$ its Killing form. Suppose that $\mathfrak{r}$ is a reductive subalgebra of $\mathfrak{g}$ such that the restriction of $B$ to $\mathfrak{r}$ is nondegenerate. Let $\mathfrak{s}$ be the orthogonal complement of $\mathfrak{r}$ with respect to $B$. Then the restriction of $B$ to $\mathfrak{s}$ is nondegenerate and we have a decomposition

$$
\mathfrak{g}=\mathfrak{r} \oplus \mathfrak{s} .
$$

Let $C(\mathfrak{s})$ be the Clifford algebra of $\mathfrak{s}$. Unlike in HP1, we will use the same defining relations as Kostant, namely

$$
v w+w v=2 B(v, w)
$$


in particular, if $B(v, v)=1$, then $v^{2}=1$, and not -1 as in [HP1]. Of course, over $\mathbb{C}$ there is no substantial difference between the two conventions. Here, as in [K2], the exterior algebra $\bigwedge(\mathfrak{s})$ is identified with the Clifford algebra $C(\mathfrak{s})$ as a vector space via Chevalley identification. We assume that $\operatorname{dim} \mathfrak{s}$ is even, because it will be the case in the applications we consider later in the paper. We denote by $S_{\mathfrak{s}}$, or simply $S$ if the context is clear, the spin module for $C(\mathfrak{s})$. As in [K4] or [K3] we identify $S$ with $\Lambda \mathfrak{s}^{+}$, where $\mathfrak{s}^{+}$is a maximal isotropic subspace of $\mathfrak{s}$.

Let $W_{1}, \ldots, W_{l}$ be an orthonormal basis of $\mathfrak{s}$. Then Kostant's cubic Dirac operator $D(\mathfrak{g}, \mathfrak{r})$ is the element

$$
D(\mathfrak{g}, \mathfrak{r})=\sum_{k} W_{k} \otimes W_{k}-\frac{1}{2} \sum_{i<j<k} B\left(\left[W_{i}, W_{j}\right], W_{k}\right) \otimes W_{i} W_{j} W_{k}
$$

of $U(\mathfrak{g}) \otimes C(\mathfrak{s})$.

One can easily see that this is in fact independent of the choice of the basis $W_{1}, \ldots, W_{l}$. Kostant's original definition uses exterior multiplication instead of Clifford multiplication in the second sum. For orthogonal vectors, there is, however, no difference between exterior and Clifford multiplication, so the above definition is the same as Kostant's.

There is a general construction we need to recall here for further references, which is the diagonal embedding of $U(\mathfrak{r})$ in $U(\mathfrak{g}) \otimes C(\mathfrak{s})$. First we embed $\mathfrak{r}$ in $U(\mathfrak{g}) \otimes C(\mathfrak{s})$ via

$$
X \longmapsto X \otimes 1+1 \otimes \alpha(X), \quad X \in \mathfrak{r},
$$

where $\alpha: \mathfrak{r} \rightarrow \mathfrak{s o}(\mathfrak{s}) \rightarrow C(\mathfrak{s})$ is the action map followed by the standard inclusion of $\mathfrak{s o}(\mathfrak{s})$ into $C(\mathfrak{s})$ using the identification $\mathfrak{s o}(\mathfrak{s}) \cong \Lambda^{2} \mathfrak{s}$. This embedding is then extended naturally to a morphism of (super)algebras:

$$
\Delta: U(\mathfrak{r}) \rightarrow U(\mathfrak{g}) \otimes C(\mathfrak{s}) .
$$

There is an explicit expression for $\alpha: \mathfrak{r} \rightarrow C(\mathfrak{s}):$ If $W_{r}$ is a basis of $\mathfrak{s}$ with dual basis $W_{r}^{*}$, then

$$
\alpha(X)=-\frac{1}{4} \sum_{r, s} B\left(\left[W_{r}^{*}, W_{s}^{*}\right], X\right) W_{r} W_{s}
$$

In this paper, we will use two instances of these general definitions. The first one is the original Dirac operator of Parthasarathy $\mathrm{P}$. Here, we assume that $G$ is a connected semisimple Lie group with finite center, and with Lie algebra $\mathfrak{g}_{0}$. Let $\theta$ be a Cartan involution of $G$, which gives Cartan decompositions

$$
\mathfrak{g}_{0}=\mathfrak{k}_{0} \oplus \mathfrak{p}_{0}, \quad G=K \exp \mathfrak{p}_{0}
$$

of $\mathfrak{g}_{0}$ and $G$. The group $K=G^{\theta}$ is a maximal compact subgroup of $G$, with Lie algebra $\mathfrak{k}_{0}$. The group $K$ maps to $\mathrm{SO}\left(\mathfrak{p}_{0}\right)$ through the adjoint action. Let us denote by $\tilde{K}$ its spin-cover. Thus, we get a canonical morphism $\tilde{K} \rightarrow \operatorname{Spin}\left(\mathfrak{p}_{0}\right)$. Since $\operatorname{Spin}\left(\mathfrak{p}_{0}\right)$ can be realized in $C(\mathfrak{p})^{\times}$, we see that $\tilde{K}$ acts canonically on $S_{\mathfrak{p}}$.

The Killing form $B$ is negative definite on $\mathfrak{k}_{0}$, and positive definite on $\mathfrak{p}_{0}$. Taking the complexification of these vector spaces, we get a decomposition $\mathfrak{g}=\mathfrak{k} \oplus \mathfrak{p}$. The construction above with $\mathfrak{r}=\mathfrak{k}$ and $\mathfrak{s}=\mathfrak{p}$ gives a Dirac operator $D(\mathfrak{g}, \mathfrak{k})$. Notice that if $X, Y, Z$ are in $\mathfrak{p},[X, Y]$ is in $\mathfrak{k}$, so $B([X, Y], Z)=0$. Thus, the second sum in (1) (the cubic part) vanishes. 
The second kind of cubic Dirac operator we consider arises in the following setting. Suppose that $\mathfrak{q}=\mathfrak{l} \oplus \mathfrak{u}$ is a parabolic subalgebra of $\mathfrak{g}$, that $\mathfrak{q}^{-}=\mathfrak{l} \oplus \mathfrak{u}^{-}$is the opposite parabolic subalgebra, and set $\mathfrak{s}=\mathfrak{u} \oplus \mathfrak{u}^{-}$. Then

$$
\mathfrak{g}=\mathfrak{l} \oplus \mathfrak{s} .
$$

Furthermore, the restrictions of the Killing form $B$ to $\mathfrak{l}$ and $\mathfrak{s}$ are nondegenerate, and the above decomposition is orthogonal. Thus, we can form the cubic Dirac operator $D(\mathfrak{g}, \mathfrak{l})$ as above. It will be convenient to use a different kind of basis of $\mathfrak{s}$ to express this operator. Indeed, since $\mathfrak{u}$ and $\mathfrak{u}^{-}$are isotropic subspaces in perfect duality under $B$, we can identify $\mathfrak{u}^{*}$ with $\mathfrak{u}^{-}$; this identification is $\mathfrak{l}$-invariant. Let $u_{1}, \ldots, u_{n}$ be a basis of $\mathfrak{u}$, and let $u_{1}^{-}, \ldots, u_{n}^{-}$be the dual basis of $\mathfrak{u}^{-}$. Consider the following dual bases of $\mathfrak{s}$ :

$$
\begin{gathered}
b_{1}=u_{1}, \ldots, b_{n}=u_{n}, b_{n+1}=u_{1}^{-}, \ldots, b_{2 n}=u_{n}^{-}, \\
d_{1}=u_{1}^{-}, \ldots, d_{n}=u_{n}^{-}, d_{n+1}=u_{1}, \ldots, d_{2 n}=u_{n} .
\end{gathered}
$$

We can now write Kostant's cubic Dirac operator $D=D(\mathfrak{g}, \mathfrak{l})$ as

$$
D=\sum_{i=1}^{2 n} d_{i} \otimes b_{i}+1 \otimes v
$$

where $v$ is the cubic element

$$
v=-\frac{1}{2} \sum_{1 \leq i<j<k \leq 2 n} B\left(\left[d_{i}, d_{j}\right], d_{k}\right) b_{i} \wedge b_{j} \wedge b_{k} .
$$

It is clear that the first sum in the expression for $D$ breaks up into two pieces:

$$
\sum_{i=1}^{n} u_{i}^{-} \otimes u_{i}+\sum_{i=1}^{n} u_{i} \otimes u_{i}^{-}=A+A^{-},
$$

where $A$, respectively $A^{-}$, denote the two summands. Moreover, since for any $i, j, k$,

$$
B\left(\left[u_{i}, u_{j}\right], u_{k}\right)=B\left(\left[u_{i}^{-}, u_{j}^{-}\right], u_{k}^{-}\right)=0,
$$

the cubic element $v$ also splits into two parts, as

$$
\begin{aligned}
v= & -\frac{1}{2} \sum_{i<j} \sum_{k} B\left(\left[u_{i}^{-}, u_{j}^{-}\right], u_{k}\right) u_{i} \wedge u_{j} \wedge u_{k}^{-} \\
& -\frac{1}{2} \sum_{i<j} \sum_{k} B\left(\left[u_{i}, u_{j}\right], u_{k}^{-}\right) u_{i}^{-} \wedge u_{j}^{-} \wedge u_{k}=a+a^{-},
\end{aligned}
$$

where $a$, respectively $a^{-}$, denote the two summands. Using equation (1.6) from K2 one can pass from exterior multiplication to Clifford multiplication and get

$$
a=-\frac{1}{4} \sum_{i, j}\left[u_{i}^{-}, u_{j}^{-}\right] u_{i} u_{j}=-\frac{1}{4} \sum_{i, j} u_{i} u_{j}\left[u_{i}^{-}, u_{j}^{-}\right]
$$

Similarly,

$$
a^{-}=-\frac{1}{4} \sum_{i, j}\left[u_{i}, u_{j}\right] u_{i}^{-} u_{j}^{-}=-\frac{1}{4} \sum_{i, j} u_{i}^{-} u_{j}^{-}\left[u_{i}, u_{j}\right] .
$$

In performing the calculation, one has to deal with expressions such as $\sum_{i, j} B\left(\left[u_{i}^{-}, u_{j}^{-}\right], u_{i}\right) u_{j}$. This expression is, however, zero, since it is an l-invariant 
element of $\mathfrak{u}$, and there are no nonzero l-invariants in $\mathfrak{u}$. For future reference, let us write the decomposition of $D$ we obtained as

$$
D=A+A^{-}+1 \otimes v=A+A^{-}+1 \otimes a+1 \otimes a^{-}=C+C^{-},
$$

where $C, C^{-}$are the following elements of $U(\mathfrak{g}) \otimes C(\mathfrak{s})$ :

$$
C=A+1 \otimes a \quad \text { and } \quad C^{-}=A^{-}+1 \otimes a^{-} .
$$

We are interested in the action of these elements on the $U(\mathfrak{g}) \otimes C(\mathfrak{s})$-module $V \otimes S$, where $V$ is a $\mathfrak{g}$-module, and $S$ is the spin module for the Clifford algebra $C(\mathfrak{s})$. As mentioned above, we use the identification of $S$ with $\Lambda \mathfrak{u}$, given explicitly in K4 and [K3]. Namely, one can construct $S$ as the left ideal in $C(\mathfrak{s})$ generated by the element $u_{\text {top }}^{-}=u_{1}^{-} \ldots u_{n}^{-}$. One then has $S=(\bigwedge \mathfrak{u}) u_{\text {top }}^{-}$, which is isomorphic to $\wedge \mathfrak{u}$ as a vector space, and the action of $C(\mathfrak{s})$ is given by left Clifford multiplication. Explicitly, $u \in \mathfrak{u}$ and $u^{-} \in \overline{\mathfrak{u}}$ act on $Y=Y_{1} \wedge \cdots \wedge Y_{p} \in \wedge^{p} \mathfrak{u}$ by

$$
\begin{gathered}
u \cdot Y=u \wedge Y ; \\
u^{-} \cdot Y=2 \sum_{i=1}^{p}(-1)^{i+1} B\left(u^{-}, Y_{i}\right) Y_{1} \wedge \cdots \wedge \hat{Y}_{i} \wedge \cdots \wedge Y_{p} .
\end{gathered}
$$

Namely, since $\mathfrak{u}$ and $\mathfrak{u}^{-}$are isotropic, the Clifford and wedge products coincide on each of them; in particular, $u^{-} u_{\text {top }}^{-}=0$.

It is quite clear that the action of $C^{-}$on $V \otimes S=V \otimes \bigwedge \mathfrak{u}$ is -2 times the action of the well-known $\mathfrak{u}$-homology differential $\partial$, which is given by

$$
\begin{aligned}
\partial\left(v \otimes Y_{1} \wedge \ldots \wedge\right. & \left.\wedge Y_{p}\right)=\sum_{i=1}^{p}(-1)^{i} Y_{i} \cdot v \otimes Y_{1} \wedge \ldots \wedge \hat{Y}_{i} \wedge \ldots \wedge Y_{p} \\
& +\sum_{1 \leq i<j \leq p}(-1)^{i+j} v \otimes\left[Y_{i}, Y_{j}\right] \wedge Y_{1} \wedge \ldots \wedge \hat{Y}_{i} \wedge \ldots \wedge \hat{Y}_{j} \wedge \ldots \wedge Y_{p}
\end{aligned}
$$

on $V \otimes \bigwedge^{p} \mathfrak{u}$. Namely, acting on a typical basis element $v \otimes u_{i_{1}} \wedge \cdots \wedge u_{i_{k}}, u_{i} \otimes u_{i}^{-}$ will produce zero if $i$ is different from all $i_{j}$, and it will produce $2(-1)^{j+1} u_{i_{j}} v \otimes$ $u_{i_{1}} \wedge \cdots \wedge \hat{u}_{i_{j}} \wedge \cdots \wedge u_{i_{k}}$ if $i=i_{j}$. Moreover, $\left[u_{i}, u_{j}\right] u_{i}^{-} u_{j}^{-}$will act as zero unless both $i$ and $j$ appear among $i_{1}, \ldots, i_{k}$, and if $i$ and $j$ do appear, then $u_{i}$ and $u_{j}$ get contracted while the commutator gets inserted, exactly as in the formula for $\partial$.

To understand the action of $C$, we first make the following identifications:

$$
V \otimes \bigwedge^{p} \mathfrak{u} \cong \operatorname{Hom}\left(\left(\bigwedge^{p} \mathfrak{u}\right)^{*}, V\right) \cong \operatorname{Hom}\left(\bigwedge^{p}\left(\mathfrak{u}^{*}\right), V\right) \cong \operatorname{Hom}\left(\bigwedge^{p} \mathfrak{u}^{-}, V\right) .
$$

The last space is the space of $p$-cochains for the $\mathfrak{u}^{-}$-cohomology differential $d$, given by the usual formula

$$
\begin{aligned}
(d \omega)\left(X_{0} \wedge\right. & \left.\ldots \wedge X_{p}\right)=\sum_{i=0}^{p}(-1)^{i} X_{i} \cdot \omega\left(X_{0} \wedge \ldots \wedge \hat{X}_{i} \wedge \ldots \wedge X_{p}\right) \\
& +\sum_{0 \leq i<j \leq p}(-1)^{i+j} \omega\left(\left[X_{i}, X_{j}\right] \wedge X_{0} \wedge \ldots \wedge \hat{X}_{i} \wedge \ldots \wedge \hat{X}_{j} \wedge \ldots \wedge X_{p}\right) .
\end{aligned}
$$

The following lemma will help us understand the action of $C$. The proof is a straightforward calculation, starting from the fact that the identification $\wedge^{p}\left(\mathfrak{u}^{*}\right)=$ $\left(\wedge^{p} \mathfrak{u}\right)^{*}$ is given via $\left(f_{1} \wedge \cdots \wedge f_{p}\right)\left(X_{1} \wedge \cdots \wedge X_{p}\right)=\operatorname{det} f_{i}\left(X_{j}\right)$. 
Lemma 2.1. Through the above identifications, the differential $d: V \otimes \bigwedge^{p} \mathfrak{u} \rightarrow$ $V \otimes \bigwedge^{p+1} \mathfrak{u}$ is given by

$$
\begin{aligned}
d\left(v \otimes Y_{1} \wedge \ldots \wedge Y_{p}\right)=\sum_{i=1}^{n} u_{i}^{-} & \cdot v \otimes u_{i} \wedge Y_{1} \wedge \ldots \wedge Y_{p} \\
& \quad+\frac{1}{2} \sum_{i=1}^{n} \sum_{j=1}^{p} v \otimes u_{i} \wedge Y_{1} \wedge \ldots \wedge\left[u_{i}^{-}, Y_{j}\right]_{\mathfrak{u}} \wedge \ldots \wedge Y_{p},
\end{aligned}
$$

where $\left[u_{i}^{-}, Y_{j}\right]_{\mathfrak{u}}$ denotes the projection of $\left[u_{i}^{-}, Y_{j}\right]$ on $\mathfrak{u}$.

It is now clear that the action of $A$ gives the first (single) sum in the expression for $d$. On the other hand, the element $1 \otimes a=-\frac{1}{4} \otimes \sum_{i, j} u_{i} u_{j}\left[u_{i}^{-}, u_{j}^{-}\right]$transforms $v \otimes Y_{1} \wedge \cdots \wedge Y_{p} \in V \otimes \wedge^{p} \mathfrak{u}$ into

$$
\begin{aligned}
& -\frac{1}{4} v \otimes \sum_{i, j} u_{i} u_{j} 2 \sum_{k=1}^{p}(-1)^{k+1} B\left(\left[u_{i}^{-}, u_{j}^{-}\right], Y_{k}\right) Y_{1} \wedge \cdots \wedge \hat{Y}_{k} \wedge \cdots \wedge Y_{p} \\
& =\frac{1}{2} v \otimes \sum_{i, j, k}(-1)^{k+1} B\left(\left[u_{i}^{-}, Y_{k}\right], u_{j}^{-}\right) u_{i} \wedge u_{j} \wedge Y_{1} \wedge \cdots \wedge \hat{Y}_{k} \wedge \cdots \wedge Y_{p} .
\end{aligned}
$$

Now we sum $\sum_{j} B\left(\left[u_{i}^{-}, Y_{k}\right], u_{j}^{-}\right) u_{j}=\left[u_{i}^{-}, Y_{k}\right]_{\mathfrak{u}}$, and after commuting $\left[u_{i}^{-}, Y_{k}\right]_{\mathfrak{u}}$ into its proper place, we get the second (double) sum in the expression for $d$.

So we see that $D=C+C^{-}$, where $C$ (resp. $C^{-}$) acts on $V \otimes S=V \otimes \wedge \mathfrak{u}$ as the $\mathfrak{u}^{-}$-cohomology differential $d$ (resp. up to a factor -2 , as the $\mathfrak{u}$-homology differential $\partial$ ). To compare the l-actions under this identification, note that the natural action of $\mathfrak{l}$ on $V \otimes S$ is the tensor product of the restriction of the $\mathfrak{g}$-action on $V$ and the spin action on $S$. On the other hand, the usual $\mathfrak{l}$ action on $\mathfrak{u}^{-}$-cohomology and $\mathfrak{u}$ homology is given by the adjoint action on $\bigwedge^{\prime} \mathfrak{u}^{-}$and $\Lambda \mathfrak{u}$. Thus, our identification of $V \otimes \bigwedge \mathfrak{u}$ with $V \otimes S$ is not an l-isomorphism. However, as was proved in [K3], Proposition 3.6, the two actions differ only by a twist with the one-dimensional l-module $Z_{-\rho(\mathfrak{u})}$ of weight $-\rho(\mathfrak{u})$.

Remark 2.2. The fact that $C$ and $C^{-}$act on $V \otimes S$ as differentials is by no means an accident as they, in fact, square to zero in $U(\mathfrak{g}) \otimes C(\mathfrak{s})$. A simple direct way of seeing this uses Kostant's formula [K2], Theorem 2.16 for the square of $D$ :

$$
D^{2}=\Omega_{\mathfrak{g}} \otimes 1-\Delta\left(\Omega_{\mathfrak{l}}\right)+c .
$$

Here $\Omega_{\mathfrak{g}}\left(\right.$ resp. $\left.\Omega_{\mathfrak{l}}\right)$ denotes the Casimir element of $Z(\mathfrak{g}) \subset U(\mathfrak{g})($ resp. $Z(\mathfrak{l}) \subset U(\mathfrak{l}))$ and $\Delta$ is the diagonal embedding

$$
\Delta: U(\mathfrak{l}) \rightarrow U(\mathfrak{g}) \otimes C(\mathfrak{s})
$$

defined in (2). Finally, $c$ is the constant $\|\rho\|^{2}-\left\|\rho_{\mathrm{r}}\right\|^{2}$.

It follows from this expression for $D^{2}$ that $D^{2}$ commutes with all l-invariant elements of $U(\mathfrak{g}) \otimes C(\mathfrak{s})$. In particular, $D^{2}$ commutes with $E=-\frac{1}{2} \sum_{i} 1 \otimes u_{i}^{*} u_{i}$. An easy direct calculation shows that

$$
[E, C]=C ; \quad\left[E, C^{-}\right]=-C^{-} .
$$

Thus,

$$
0=\left[E, D^{2}\right]=\left[E, C^{2}+C C^{-}+C^{-} C+\left(C^{-}\right)^{2}\right]=2 C^{2}-2\left(C^{-}\right)^{2},
$$


hence $C^{2}=\left(C^{-}\right)^{2}$. Combined with $\left[E, C^{2}\right]=2 C^{2}$ and $\left[E,\left(C^{-}\right)^{2}\right]=-2\left(C^{-}\right)^{2}$ this implies $C^{2}=\left(C^{-}\right)^{2}=0$.

Remark 2.3. One can prove a result for $C$ and $C^{-}$analogous to the one obtained for $D$ in [HP1] and [K4]. Namely, let $d_{C}$ and $d_{C^{-}}$be operators on $U(\mathfrak{g}) \otimes C(\mathfrak{s})$ defined by supercommuting with $C$, respectively $C^{-}$. Then both $d_{C}$ and $d_{C^{-}}$are l-equivariant, and they define differentials on the algebra of l-invariants in $U(\mathfrak{g}) \otimes C(\mathfrak{s})$. These differentials both have cohomology $Z\left(\mathfrak{l}_{\Delta}\right)$. More precisely,

$$
\text { Ker } d_{C}=Z\left(\mathfrak{l}_{\Delta}\right) \oplus \operatorname{Im} d_{C} \quad \text { and } \quad \operatorname{Ker} d_{C^{-}}=Z\left(\mathfrak{l}_{\Delta}\right) \oplus \operatorname{Im} d_{C^{-}} .
$$

Using this and some additional computations, one can obtain the well-known Casselman-Osborne theorem as a corollary. This sheds some light on the formal similarity between the Casselman-Osborne theorem and Vogan's conjecture proved in [HP1].

\section{Relative Dirac operators}

In this section we compare various Dirac operators arising from two compatible decompositions

$$
\mathfrak{g}=\mathfrak{l} \oplus \mathfrak{s}=\mathfrak{k} \oplus \mathfrak{p}
$$

as in the previous section. More precisely, we assume that $G$ is a semi-simple Lie group, with Cartan involution $\theta$. We use the notation of the previous section for this setting. Futhermore, we assume that $\mathfrak{q}=\mathfrak{l} \oplus \mathfrak{u}$ is a $\theta$-stable parabolic subalgebra of $\mathfrak{g}$, with $\mathfrak{l} \subset \mathfrak{k}$. This implies, in particular, that $G$ and $K$ have the same rank. Since $\mathfrak{u}$ is $\theta$-stable, we have $\mathfrak{u}=\mathfrak{u}_{\mathfrak{k}} \oplus \mathfrak{u}_{\mathfrak{p}}$ where $\mathfrak{u}_{\mathfrak{k}}=\mathfrak{u} \cap \mathfrak{k}$ and $\mathfrak{u}_{\mathfrak{p}}=\mathfrak{u} \cap \mathfrak{p}$. In the same way, we write $\mathfrak{u}^{-}=\mathfrak{u}_{\mathfrak{k}}^{-} \oplus \mathfrak{u}_{\mathfrak{p}}^{-}$and $\mathfrak{s}=\mathfrak{s}_{\mathfrak{k}} \oplus \mathfrak{s}_{\mathfrak{p}}$.

We have attached Dirac operators $D(\mathfrak{g}, \mathfrak{k}), D(\mathfrak{g}, \mathfrak{l})$ and $D(\mathfrak{k}, \mathfrak{l})$ to these decompositions. Our goal is now to relate these operators, but they live respectively in $U(\mathfrak{g}) \otimes C(\mathfrak{s}), U(\mathfrak{g}) \otimes C(\mathfrak{p})$ and $U(\mathfrak{k}) \otimes C(\mathfrak{s} \mathfrak{k})$. To be able to compare them, we first write

$$
C(\mathfrak{s})=C(\mathfrak{p}) \bar{\otimes} C\left(\mathfrak{s}_{\mathfrak{k}}\right),
$$

where $\bar{\otimes}$ denotes the $\mathbb{Z}_{2}$-graded tensor product. In view of this, we consider $U(\mathfrak{g}) \otimes$ $C(\mathfrak{p})$ as the subalgebra $U(\mathfrak{g}) \otimes(C(\mathfrak{p}) \bar{\otimes} 1)$ of $U(\mathfrak{g}) \otimes C(\mathfrak{s})$. We will now consider elements of $U(\mathfrak{g}) \otimes C(\mathfrak{p})$ such as $D(\mathfrak{g}, \mathfrak{k})$ as elements of $U(\mathfrak{g}) \otimes C(\mathfrak{s})$ without further notice. Similarly, we use the diagonal embedding

$$
\Delta: U(\mathfrak{k}) \hookrightarrow U(\mathfrak{g}) \otimes C(\mathfrak{p})
$$

defined in (2) to get an embedding

$$
\Delta: U(\mathfrak{k}) \otimes C\left(\mathfrak{s}_{\mathfrak{k}}\right) \hookrightarrow U(\mathfrak{g}) \otimes\left(C(\mathfrak{p}) \bar{\otimes} C\left(\mathfrak{s}_{\mathfrak{k}}\right)\right) \cong U(\mathfrak{g}) \otimes C(\mathfrak{s}) .
$$

Let $u_{1}, \ldots, u_{k}$ be a basis for $\mathfrak{u}_{\mathfrak{k}}$ and let $v_{1}, \ldots, v_{p}$ be a basis for $\mathfrak{u}_{\mathfrak{p}}$ with dual bases, respectively, $u_{1}^{-}, \ldots, u_{k}^{-}$of $\mathfrak{u}_{\mathfrak{k}}^{-}$and $v_{1}^{-}, \ldots, v_{p}^{-}$of $\mathfrak{u}_{\mathfrak{p}}^{-}$. As before, $D=D(\mathfrak{g}, \mathfrak{l})$, $C=C(\mathfrak{g}, \mathfrak{l})=A+1 \otimes a$ and $C^{-}=C^{-}(\mathfrak{g}, \mathfrak{l})=A^{-}+1 \otimes a^{-}$denote the Dirac operator for the pair $(\mathfrak{g}, \mathfrak{l})$ and its parts. We can now further decompose these parts and write

$$
A=A_{\mathfrak{k}}+A_{\mathfrak{p}} ; \quad a=a_{\mathfrak{k}}+a_{\mathfrak{k}}+a_{\mathfrak{p}}
$$


and analogously for $A^{-}$and $a^{-}$. Here

$$
\begin{gathered}
A_{\mathfrak{k}}=\sum_{i} u_{i}^{-} \otimes u_{i}, \quad A_{\mathfrak{p}}=\sum_{i} v_{i}^{-} \otimes v_{i} ; \\
a_{\mathfrak{k}}=-\frac{1}{4} \sum_{i, j}\left[u_{i}^{-}, u_{j}^{-}\right] u_{i} u_{j}, \quad a_{\mathfrak{k}}=-\frac{1}{2} \sum_{i, j}\left[u_{i}^{-}, v_{j}^{-}\right] u_{i} v_{j}, \quad a_{\mathfrak{p}}=-\frac{1}{4} \sum_{i, j}\left[v_{i}^{-}, v_{j}^{-}\right] v_{i} v_{j} .
\end{gathered}
$$

The expressions for $A_{\mathfrak{k}}^{-}, A_{\mathfrak{p}}^{-}, a_{\mathfrak{k}}^{-}, a_{\mathfrak{k} \mathfrak{p}}^{-}$and $a_{\mathfrak{p}}^{-}$are obtained by exchanging $u_{i}$ with $u_{i}^{-}$and $v_{i}$ with $v_{i}^{-}$.

In the following, we will consider the Clifford algebra $C(\mathfrak{s})$ as a subalgebra of $U(\mathfrak{g}) \otimes C(\mathfrak{s})$, embedded as $1 \otimes C(\mathfrak{s})$. In particular, $1 \otimes a_{\mathfrak{k}}$ gets identified with $a_{\mathfrak{k}}$, $1 \otimes a_{\mathfrak{k} \mathfrak{p}}$ with $a_{\mathfrak{k p}}$ and so on.

Clearly, since $\mathfrak{p}=\mathfrak{u}_{\mathfrak{p}} \oplus \mathfrak{u}_{\mathfrak{p}}^{-}$, we see that $D(\mathfrak{g}, \mathfrak{k})=A_{\mathfrak{p}}+A_{\mathfrak{p}}^{-}$. In the obvious notation, $D(\mathfrak{k}, \mathfrak{l})=C(\mathfrak{k}, \mathfrak{l})+C^{-}(\mathfrak{k}, \mathfrak{l})$, and we want now to identify the images of these elements under $\Delta$. Denote these images by $D_{\Delta}(\mathfrak{k}, \mathfrak{l}), C_{\Delta}(\mathfrak{k}, \mathfrak{l})$ and $C_{\Delta}^{-}(\mathfrak{k}, \mathfrak{l})$. To do this, we use the expression (3) for $\alpha: \mathfrak{k} \rightarrow C(\mathfrak{p})$ with respect to the basis $v_{1}, \ldots, v_{p}, v_{1}^{-}, \ldots, v_{p}^{-}$and the dual basis $v_{1}^{-}, \ldots, v_{p}^{-}, v_{1}, \ldots, v_{p}$. We get

$$
\begin{aligned}
\alpha(X)= & -\frac{1}{4} \sum_{j, k} B\left(\left[v_{j}^{-}, v_{k}^{-}\right], X\right) v_{j} v_{k}-\frac{1}{2} \sum_{j, k} B\left(\left[v_{j}, v_{k}^{-}\right], X\right) v_{j}^{-} v_{k} \\
& +\frac{1}{2} \sum_{j} B\left(\left[v_{j}, v_{j}^{-}\right], X\right)-\frac{1}{4} \sum_{j, k} B\left(\left[v_{j}, v_{k}\right], X\right) v_{j}^{-} v_{k}^{-}
\end{aligned}
$$

(we used $v_{j}^{-} v_{k}=-v_{k} v_{j}^{-}+2 \delta_{j k}$ ).

Since $C(\mathfrak{k}, \mathfrak{l})=\sum_{i} u_{i}^{-} \otimes u_{i}-\frac{1}{4} \otimes \sum_{i, j}\left[u_{i}^{-}, u_{j}^{-}\right] u_{i} u_{j}$, we see that

$$
\Delta(C(\mathfrak{k}, \mathfrak{l}))=A_{\mathfrak{k}}+\sum_{i} 1 \otimes \alpha\left(u_{i}^{-}\right) \otimes u_{i}+a_{\mathfrak{k}} .
$$

We need to calculate the middle term, $\sum_{i} 1 \otimes \alpha\left(u_{i}^{-}\right) \otimes u_{i}$. Applying the above expression for $\alpha$, we get four sums over $i, j$ and $k$.

We first notice that the first of these four sums is 0 , since $B$ is 0 on $\mathfrak{u}^{-}$. To calculate the second sum, write $B\left(\left[v_{j}, v_{k}^{-}\right], u_{i}^{-}\right)=B\left(v_{j},\left[v_{k}^{-}, u_{i}^{-}\right]\right)$, and observe that since $\left[v_{k}^{-}, u_{i}^{-}\right] \in \mathfrak{u}^{-} \cap \mathfrak{p}, \sum_{j} B\left(v_{j},\left[v_{k}^{-}, u_{i}^{-}\right]\right) v_{j}^{-}=\left[v_{k}^{-}, u_{i}^{-}\right]$. Therefore, the second of the four sums is

$$
-\frac{1}{2} \otimes \sum_{i, k}\left[v_{k}^{-}, u_{i}^{-}\right] v_{k} u_{i}=a_{\mathfrak{k p}} .
$$

The third sum is 0 , since we can assume $\left[v_{j}, v_{j}^{-}\right]$is in $\mathfrak{l}$ and hence orthogonal to $u_{i}^{-}$. Namely, we can choose $v_{j}$ and $v_{j}^{-}$(and also $u_{i}$ and $u_{i}^{-}$) to be root vectors with respect to a Cartan subalgebra $\mathfrak{t}$ of $\mathfrak{l}$.

Finally, the fourth sum is calculated by noting that since $\left[v_{j}, v_{k}\right] \in \mathfrak{u} \cap \mathfrak{k}$,

$$
\sum_{i} B\left(\left[v_{j}, v_{k}\right], u_{i}^{-}\right) u_{i}=\left[v_{j}, v_{k}\right]
$$

It follows that the fourth sum is

$$
-\frac{1}{4} \otimes \sum_{j, k} v_{j}^{-} v_{k}^{-}\left[v_{j}, v_{k}\right]=a_{\mathfrak{p}}^{-} .
$$

A completely analogous calculation applies to $C^{-}(\mathfrak{k}, \mathfrak{l})$, so we proved: 
Proposition 3.1. Under the diagonal map

$$
\Delta: U(\mathfrak{k}) \otimes C\left(\mathfrak{s}_{\mathfrak{k}}\right) \rightarrow U(\mathfrak{g}) \otimes C(\mathfrak{p}) \bar{\otimes} C\left(\mathfrak{s}_{\mathfrak{k}}\right),
$$

$C(\mathfrak{k}, \mathfrak{l})$ and $C^{-}(\mathfrak{k}, \mathfrak{l})$ correspond to

$$
C_{\Delta}(\mathfrak{k}, \mathfrak{l})=A_{\mathfrak{k}}+a_{\mathfrak{k}}+a_{\mathfrak{k} \mathfrak{p}}+a_{\mathfrak{p}}^{-} \quad \text { and } \quad C_{\Delta}^{-}(\mathfrak{k}, \mathfrak{l})=A_{\mathfrak{k}}^{-}+a_{\mathfrak{k}}^{-}+a_{\mathfrak{k} \mathfrak{p}}^{-}+a_{\mathfrak{p}} .
$$

Note the unexpected feature of this result, the mixing of the positive and negative parts under the diagonal embedding. Namely, $a_{\mathfrak{p}}$ and $a_{\mathfrak{p}}^{-}$have opposite positions from the ones one would expect. So, we get:

Theorem 3.2. With notation as above,

(i) $D(\mathfrak{g}, \mathfrak{l})=D(\mathfrak{g}, \mathfrak{k})+D_{\Delta}(\mathfrak{k}, \mathfrak{l})$;

(ii) the summands $D(\mathfrak{g}, \mathfrak{k})$ and $D_{\Delta}(\mathfrak{k}, \mathfrak{l})$ anticommute.

Proof. We have seen that

$$
\begin{aligned}
D(\mathfrak{g}, \mathfrak{l}) & =A+A^{-}+a+a^{-} \\
& =A_{\mathfrak{k}}+A_{\mathfrak{p}}+A_{\mathfrak{k}}^{-}+A_{\mathfrak{p}}^{-}+a_{\mathfrak{k}}+a_{\mathfrak{k} \mathfrak{p}}+a_{\mathfrak{p}}+a_{\mathfrak{k}}^{-}+a_{\mathfrak{k p}}^{-}+a_{\mathfrak{p}}^{-} \\
& =\left(A_{\mathfrak{p}}+A_{\mathfrak{p}}^{-}\right)+\left(A_{\mathfrak{k}}+a_{\mathfrak{k}}+a_{\mathfrak{k p}}+a_{\mathfrak{p}}^{-}\right)+\left(A_{\mathfrak{k}}^{-}+a_{\mathfrak{k}}^{-}+a_{\mathfrak{k p}}^{-}+a_{\mathfrak{p}}\right) \\
& =D(\mathfrak{g}, \mathfrak{k})+C_{\Delta}(\mathfrak{k}, \mathfrak{l})+C_{\Delta}^{-}(\mathfrak{k}, \mathfrak{l}),
\end{aligned}
$$

which proves (i).

To prove (ii), we use the fact that $D(\mathfrak{g}, \mathfrak{k})$ commutes with $\mathfrak{k}_{\Delta}$, which is one of the most basic properties of $D(\mathfrak{g}, \mathfrak{k})$. It follows that for $Z \in \mathfrak{s} \mathfrak{k}$, the anticommutator

$$
[D(\mathfrak{g}, \mathfrak{k}) \otimes 1,(Z \otimes 1+1 \otimes \alpha(Z)) \otimes Z]=[D(\mathfrak{g}, \mathfrak{k}), Z \otimes 1+1 \otimes \alpha(Z)] \otimes Z
$$

is zero. Hence $[D(\mathfrak{g}, \mathfrak{k}) \otimes 1, \Delta(Z \otimes Z)]=0$. From the formula (11) for $D(\mathfrak{k}, \mathfrak{l})$, we see that it remains to be checked that for an orthonormal basis $\left(Z_{i}\right)_{i}$ of $\mathfrak{s}_{k}$,

$$
\left[D(\mathfrak{g}, \mathfrak{r}) \otimes 1,1 \otimes 1 \otimes \sum_{i<j<k} B\left(\left[Z_{i}, Z_{j}\right], Z_{k}\right) Z_{i} Z_{j} Z_{k}\right]=0 .
$$

(Here the computation takes place in $U(\mathfrak{g}) \otimes C(\mathfrak{p}) \bar{\otimes} C(\mathfrak{s} \mathfrak{k})$.) This follows from the definition of $\bar{\otimes}$, since all the $C(\mathfrak{p})$-parts of the monomial terms of $D(\mathfrak{g}, \mathfrak{k})$, and also all $Z_{i} Z_{j} Z_{k} \in C\left(\mathfrak{s}_{\mathfrak{k}}\right)$, are odd.

\section{HeRmitian Forms AND ADJOINTS}

Let $\mathfrak{r}$ be a subalgebra of $\mathfrak{g}$ to which $B$ restricts nondegenerately and let $\mathfrak{s}$ be the orthogonal complement to $\mathfrak{r}$ with respect to $B$. We choose a maximal isotropic subspace $\mathfrak{s}^{+}$of $\mathfrak{s}$. Since

$$
\langle X, Y\rangle=-2 B(X, \theta \bar{Y})
$$

(with ${ }^{-}$denoting conjugation with respect to $\mathfrak{g}_{0}$ ) defines a positive definite Hermitian form on $\mathfrak{g}$ and hence also on $\mathfrak{s}^{+}$, the subspace $\mathfrak{s}^{-}=\theta\left(\overline{\mathfrak{s}^{+}}\right)$intersects $\mathfrak{s}^{+}$trivially. Let $S=\bigwedge^{\prime} \mathfrak{s}^{+}$be a spin module for the Clifford algebra $C(\mathfrak{s})$ corresponding to this polarization. We extend the form $\langle$,$\rangle to all of S$ in the usual way, using the determinant.

Lemma 4.1. With respect to the form $\langle X, Y\rangle$ on $S$, the adjoint of the operator $X \in \mathfrak{s} \subset C(\mathfrak{s})$ is $-\theta \bar{X}$. 
Proof. We need to show that

$$
\langle X \cdot \lambda, \mu\rangle=\langle\lambda,-\theta(\bar{X}) \cdot \mu\rangle
$$

for any $X \in \mathfrak{s}$ and $\lambda, \mu \in S$. Assume $X \in \mathfrak{s}^{+}$. We can assume that $\lambda=\lambda_{1} \wedge \cdots \wedge \lambda_{s}$ and $\mu=\mu_{1} \wedge \cdots \wedge \mu_{t}$, with $X, \lambda_{i}$ and $\mu_{j}$ all belonging to a fixed orthonormal basis of $\mathfrak{s}^{+}$. Then both sides of the equality to be established are nonzero precisely when $X$ is one of the $\mu_{j}$ 's, while the other $\mu_{j}$ 's are precisely (all) the $\lambda_{i}$ 's. In this case both sides are clearly the same. If $X \in \mathfrak{s}^{-}$, the claim follows by considering $\theta(\bar{X}) \in \mathfrak{s}^{+}$.

We now assume that $\mathfrak{q}=\mathfrak{l} \oplus \mathfrak{u}$ is a $\theta$-stable parabolic subalgebra of $\mathfrak{g}$, and that $\mathfrak{l}$ is the complexification of a real Levi subalgebra $\mathfrak{l}_{0}$ of $\mathfrak{g}_{0}$. In this case, we have

$$
\mathfrak{u}^{-}=\theta(\overline{\mathfrak{u}})=\overline{\mathfrak{u}}, \quad \mathfrak{q}^{-}=\mathfrak{l} \oplus \overline{\mathfrak{u}}
$$

so the opposite parabolic subalgebra of $\mathfrak{q}$ is its complex conjugate. As in the previous section, we write $\mathfrak{u}=\mathfrak{u}_{\mathfrak{k}} \oplus \mathfrak{u}_{\mathfrak{p}}, \overline{\mathfrak{u}}=\overline{\mathfrak{u}}_{\mathfrak{k}} \oplus \overline{\mathfrak{u}}_{\mathfrak{p}}$. Let $u_{1}, \ldots, u_{k}$ be a basis of $\mathfrak{u}_{\mathfrak{k}}$ and $v_{1}, \ldots, v_{p}$ a basis of $\mathfrak{u}_{\mathfrak{p}}$. We can normalize these vectors so that the dual bases are given by $u_{1}^{-}=-\bar{u}_{1}, \ldots, u_{k}^{-}=-\bar{u}_{k}, v_{1}^{-}=\bar{v}_{1}, \ldots, v_{k}^{-}=\bar{v}_{k}$. The rest of the notation is as in the previous section.

Lemma 4.2. The cubic part $v=a+a^{-}$of Kostant's cubic Dirac operator $D=$ $D(\mathfrak{g}, \mathfrak{l})$ is self-adjoint with respect to the form $\langle$,$\rangle on S$. More precisely, a is adjoint to $a^{-}$.

Proof. This follows immediately from Lemma4.1 and the formulas for $a$ and $a^{-}$.

In the following we assume that $V$ is a unitary $(\mathfrak{g}, K)$-module. We denote by $\langle,\rangle_{V}$ the corresponding positive definite Hermitian form on $V$. Thus, every element $X$ of $\mathfrak{g}_{0}$ is skew self-adjoint with respect to $\langle,\rangle_{V}$, and for any $X \in \mathfrak{g}$, the adjoint of $X$ with respect to $\langle,\rangle_{V}$ is $-\bar{X}$. We also consider the definite Hermitian form $\langle$, on $S$ introduced above, and we denote the tensor product form on $V \otimes S$ again by $\langle$,$\rangle .$

Let us compute adjoints of elements $u_{1}, \ldots, u_{k}, v_{1}, \ldots, v_{p}$, with respect to their action on $V$ and $S$. The adjoint of the operator $u_{i}$ acting on $V$ or $S$ is $-\bar{u}_{i}=u_{i}^{-}$. The adjoint $v_{i}$ acting on $V$ is $-\bar{v}_{i}=-v_{i}^{-}$, while the adjoint of $v_{i}$ acting on $S$ is also $\bar{v}_{i}=v_{i}^{-}$. Thus, we see:

Corollary 4.3. With respect to the form $\langle$,$\rangle on V \otimes S$, the adjoint of $A_{\mathfrak{k}}$ is $A_{\mathfrak{k}}^{-}$ while the adjoint of $A_{\mathfrak{p}}$ is $-A_{\mathfrak{p}}^{-}$. Hence the adjoint of $C=A_{\mathfrak{k}}+A_{\mathfrak{p}}+1 \otimes a$ is $A_{\mathfrak{k}}^{-}+1 \otimes a^{-}-A_{\mathfrak{p}}^{-}$and the adjoint of $C^{-}=A_{\mathfrak{k}}^{-}+A_{\mathfrak{p}}^{-}+1 \otimes a^{-}$is $A_{\mathfrak{k}}+1 \otimes a-A_{\mathfrak{p}}$.

\section{Some Algebraic lemmas}

In this section, we state some easy facts from linear algebra that we will apply later. We define the cohomology of any linear operator $T$ on a vector space $V$ to be the vector space $H(T)=\operatorname{Ker} T /(\operatorname{Im} T \cap \operatorname{Ker} T)$.

Lemma 5.1. Let $A$ and $B$ be anticommuting linear operators on a vector space $V$. Assume that $A^{2}$ diagonalizes on $V$, i.e., $V=\bigoplus_{\lambda} V_{\lambda}$, with $A^{2}=\lambda \operatorname{Id}$ on $V_{\lambda}$. Then the cohomology $H(A+B)$ of $A+B$ on $V$ is the same as the cohomology of the restriction of $A+B$ to $V_{0}=\operatorname{Ker} A^{2}$. 
Proof. Since $A+B$ commutes with $A^{2}$, its kernel, image and cohomology decompose accordingly to eigenspaces $V_{\lambda}$. We thus have to prove that $A+B$ has no cohomology on $V_{\lambda}$ for $\lambda \neq 0$. In other words, we are to prove that $\operatorname{Ker}(A+B) \subset \operatorname{Im}(A+B)$ on $V_{\lambda}$.

Let $v \in V_{\lambda}$ be such that $(A+B) v=0$, i.e., $A v=-B v$. Then

$$
(A+B) A v=A^{2} v+B A v=A^{2} v-A B v=2 A^{2} v=2 \lambda v,
$$

and hence $v=\frac{1}{2 \lambda}(A+B) A v$ is in the image of $A+B$.

Corollary 5.2. In the setting above, assume further that $\operatorname{Ker} A \cap \operatorname{Im} A=0$, so $\operatorname{Ker} A^{2}=\operatorname{Ker} A=H(A)$. Then $H(A+B)$ is equal to the cohomology of $B$ restricted to the cohomology (i.e., kernel) of $A$.

Proof. By the lemma, $H(A+B)$ is the cohomology of $A+B$ on Ker $A$. But on Ker $A, A+B=B$.

Lemma 5.3. Let $A$ and $B$ be anticommuting linear operators on a vector space $V$, such that $A^{2}$ and $B$ are semisimple (i.e., can be diagonalized). Then $H(A+B)$ is the cohomology (i.e., the kernel) of $B$ acting on $H(A)$.

Proof. Applying the lemma above, we can replace $V$ by $\operatorname{Ker} A^{2}$, i.e., assume $A^{2}=0$. On the other hand, by the corollary, with the role of $A$ and $B$ exchanged, $H(A+B)$ is the cohomology of $A$ acting on $\operatorname{Ker} B$. Since $B$ is semisimple, we can decompose

$$
V=\operatorname{Ker} B \oplus \bigoplus_{\lambda} V_{\lambda} \oplus V_{-\lambda}
$$

into the (discrete) sum of eigenspaces for $B$. Here if both $\lambda$ and $-\lambda$ are eigenvalues, we choose one of them to represent the pair. Since $A$ anticommutes with $B$, it preserves $\operatorname{Ker} B$, and maps $V_{\lambda}$ to $V_{-\lambda}$ and vice versa. Therefore, $H(A)$ decomposes into a Ker $B$-part and $V_{\lambda} \oplus V_{-\lambda}$-parts. The $\operatorname{Ker} B$-part is equal to $H(A+B)$ and we will be done if we show that $B$ has no kernel on $H\left(\left.A\right|_{V_{\lambda} \oplus V_{-\lambda}}\right)$. Let $v=v_{1}+v_{2} \in$ $V_{\lambda} \oplus V_{-\lambda}$ be in $\operatorname{Ker} A$, and assume that $B v \in \operatorname{Im} A$. This implies $\lambda v_{1}-\lambda v_{2}$ is in $\operatorname{Im} A$, so $v_{1}-v_{2} \in \operatorname{Im} A$. This, however, can only happen if both $v_{1}$ and $v_{2}$ are in $\operatorname{Im} A$, again because $A$ exchanges $V_{\lambda}$ and $V_{-\lambda}$. But then also $v=v_{1}+v_{2}$ is in $\operatorname{Im} A$, so $v$ is zero in cohomology and we are done.

\section{Dirac Cohomology in stages}

If $\mathfrak{g}=\mathfrak{r} \oplus \mathfrak{s}$ is an orthogonal decomposition of $\mathfrak{g}$ with respect to the Killing form $B$, and if $V$ is a $\mathfrak{g}$-module, let us denote by $H_{D}(\mathfrak{g}, \mathfrak{r} ; V)$ the Dirac cohomology of $V$ with respect to $D(\mathfrak{g}, \mathfrak{r})$. The reader should bear in mind that $H_{D}(\mathfrak{g}, \mathfrak{r} ; V)$ is in fact the cohomology of the operator $D(\mathfrak{g}, \mathfrak{r})$ on the space $V \otimes S$.

Let us suppose we are now in the setting of Section 3 , and we adopt the notation of that section. Let $V$ be an admissible $(\mathfrak{g}, K)$-module. We can decompose $V \otimes S_{\mathfrak{p}}$ into $\tilde{K}$-isotypic components. This is a direct sum of finite-dimensional (unitary) $\tilde{K}$-modules, all of them stable under the action of $(U(\mathfrak{g}) \otimes C(\mathfrak{p}))^{\tilde{K}}$. Hence, there is a positive definite $\tilde{K}$-invariant form $\langle$,$\rangle on V \otimes S=V \otimes S_{\mathfrak{p}} \otimes S_{\mathfrak{s}_{\mathfrak{k}}}$, and from Corollary 4.3 applied to the case $\mathfrak{g}=\mathfrak{k}, D_{\Delta}(\mathfrak{k}, \mathfrak{l})$ is self-adjoint with respect to $\langle$,$\rangle . It follows$ that $B=D_{\Delta}(\mathfrak{k}, \mathfrak{l})$ is a semisimple operator, while for $A=D(\mathfrak{g}, \mathfrak{k})$ we have that $A^{2}$ is semisimple (this comes from the formula $D(\mathfrak{g}, \mathfrak{k})^{2}=\Omega_{\mathfrak{g}} \otimes 1-\Delta\left(\Omega_{\mathfrak{k}}\right)+c$, which 
in this case goes back at least to Parthasarathy $[\mathrm{P}]$ ). In this situation, we get the following result:

Theorem 6.1. Let $V$ be an admissible $(\mathfrak{g}, K)$-module. Then the Dirac cohomology with respect to $D(\mathfrak{g}, \mathfrak{l})$ can be calculated as the Dirac cohomology with respect to $D(\mathfrak{k}, \mathfrak{l})$ of the Dirac cohomology with respect to $D(\mathfrak{g}, \mathfrak{k})$ of $V$. In other words,

$$
H_{D}(\mathfrak{g}, \mathfrak{l} ; V)=H_{D}\left(\mathfrak{k}, \mathfrak{l} ; H_{D}(\mathfrak{g}, \mathfrak{k} ; V)\right) .
$$

Also, we can reverse the order of taking Dirac cohomology, i.e.,

$$
H_{D}(\mathfrak{g}, \mathfrak{l} ; V)=H\left(\left.D(\mathfrak{g}, \mathfrak{k})\right|_{H_{D}(\mathfrak{k}, \mathfrak{l} ; V)}\right) .
$$

Proof. The first formula is an application of Lemma 5.3. and the second is a direct application of Corollary 5.2 , with $A=D_{\Delta}(\mathfrak{k}, \mathfrak{r})$ and $B=D(\mathfrak{g}, \mathfrak{k})$.

\section{Hodge decomposition for $\overline{\mathfrak{u}}-\mathrm{COHOMOlOgy}$ in Hermitian CASE}

In this section, we assume that the pair $(\mathfrak{g}, \mathfrak{k})$ is Hermitian symmetric. This means that $\mathfrak{p}$ decomposes as $\mathfrak{p}=\mathfrak{p}^{+} \oplus \mathfrak{p}^{-}$, that $\mathfrak{d}=\mathfrak{k} \oplus \mathfrak{p}^{+}$is a $\theta$-stable parabolic subalgebra of $\mathfrak{g}$ with Levi factor $\mathfrak{k}$, abelian nilradical $\mathfrak{p}^{+}$, and opposite parabolic subalgebra $\mathfrak{d}^{-}=\mathfrak{k} \oplus \mathfrak{p}^{-}$. We are then in a case where the two instances of the general setting we introduced in Section 2 actually coincide. As in the previous section, let $\mathfrak{q}=\mathfrak{l} \oplus \mathfrak{u}$ be a $\theta$-stable parabolic subalgebra of $\mathfrak{g}$, with $\mathfrak{l} \subset \mathfrak{k}$ being the complexification of a real Levi subalgebra $\mathfrak{l}_{0}$ and assume furthermore that $\mathfrak{u} \supset \mathfrak{p}^{+}$.

Let us specialize in this setting the decomposition

$$
D=D(\mathfrak{g}, \mathfrak{l})=A_{\mathfrak{k}}+A_{\mathfrak{p}}+A_{\mathfrak{k}}^{-}+A_{\mathfrak{p}}^{-}+a_{\mathfrak{k}}+a_{\mathfrak{k p}}+a_{\mathfrak{p}}+a_{\mathfrak{k}}^{-}+a_{\mathfrak{k} \mathfrak{p}}^{-}+a_{\mathfrak{p}}^{-}
$$

of Section 3 . We see that

$$
A_{\mathfrak{p}}=C(\mathfrak{g}, \mathfrak{k}), A_{\mathfrak{p}}^{-}=C^{-}(\mathfrak{g}, \mathfrak{k}), \quad a_{\mathfrak{p}}=a_{\mathfrak{p}}^{-}=0 .
$$

We can also extend easily the results of Theorem 3.2

Proposition 7.1. (i) $C(\mathfrak{g}, \mathfrak{l})=C(\mathfrak{g}, \mathfrak{k})+C_{\Delta}(\mathfrak{k}, \mathfrak{l}) ; C^{-}(\mathfrak{g}, \mathfrak{l})=C^{-}(\mathfrak{g}, \mathfrak{k})+C_{\Delta}^{-}(\mathfrak{k}, \mathfrak{l})$;

(ii) $\left[C(\mathfrak{g}, \mathfrak{k}), C_{\Delta}(\mathfrak{k}, \mathfrak{l})\right]=\left[C(\mathfrak{g}, \mathfrak{k}), C_{\Delta}^{-}(\mathfrak{k}, \mathfrak{l})\right]=\left[C^{-}(\mathfrak{g}, \mathfrak{k}), C_{\Delta}(\mathfrak{k}, \mathfrak{l})\right]$

$$
=\left[C^{-}(\mathfrak{g}, \mathfrak{k}), C_{\Delta}^{-}(\mathfrak{k}, \mathfrak{l})\right]=0 .
$$

Let $V$ be a unitary irreducible $(\mathfrak{g}, K)$-module, and consider the form $\langle$,$\rangle on$ $V \otimes S$ introduced in Section 4. To apply the results in this section, we decompose

$$
V \otimes S=V \otimes S_{\mathfrak{s}}=V \otimes S_{\mathfrak{p}} \otimes S_{\mathfrak{s} \cap \mathfrak{k}} \cong V \otimes \wedge \mathfrak{p}^{+} \otimes \bigwedge \mathfrak{u}_{\mathfrak{k}}
$$

By Corollary 4.3, and (5) above, the adjoints of $C_{\Delta}(\mathfrak{k}, \mathfrak{l})$ and $C(\mathfrak{g}, \mathfrak{k})$ are, respectively, $C_{\Delta}^{-}(\mathfrak{k}, \mathfrak{l})$ and $-C^{-}(\mathfrak{g}, \mathfrak{k})$. So the adjoint of $C=C(\mathfrak{g}, \mathfrak{l})$ is $C^{\text {adj }}=$ $C_{\Delta}^{-}(\mathfrak{k}, \mathfrak{l})-C^{-}(\mathfrak{g}, \mathfrak{k})$.

We consider the positive semidefinite operator $\Delta=C C^{\text {adj }}+C^{\text {adj }} C=\left[C, C^{\text {adj }}\right]$. By the proposition above

$$
\begin{aligned}
\Delta & =\left[C_{\Delta}(\mathfrak{k}, \mathfrak{l})+C(\mathfrak{g}, \mathfrak{k}), C_{\Delta}^{-}(\mathfrak{k}, \mathfrak{l})-C^{-}(\mathfrak{g}, \mathfrak{k})\right] \\
& =\left[C_{\Delta}(\mathfrak{k}, \mathfrak{l}), C_{\Delta}^{-}(\mathfrak{k}, \mathfrak{l})\right]-\left[C(\mathfrak{g}, \mathfrak{k}), C^{-}(\mathfrak{g}, \mathfrak{k})\right]=D_{\Delta}(\mathfrak{k}, \mathfrak{l})^{2}-D(\mathfrak{g}, \mathfrak{k})^{2} .
\end{aligned}
$$

We know from Corollary 4.3. applied this time to the case $\mathfrak{l}=\mathfrak{k}$, that $D(\mathfrak{g}, \mathfrak{k})$ acting on $V \otimes S_{\mathfrak{p}}$ is anti-self-adjoint. Thus, $V \otimes S_{\mathfrak{p}}$ decomposes into eigenspaces of $D(\mathfrak{g}, \mathfrak{k})^{2}$ for nonpositive eigenvalues. Each eigenspace is $\tilde{K}$-invariant, and each $\tilde{K}$-isotypic 
component of $V \otimes S_{\mathfrak{p}}$ is contained in an eigenspace. Since $V$ is admissible, the eigenspaces are finite-dimensional.

Passing from $V \otimes S_{\mathfrak{p}}$ to $V \otimes S$ is tensoring with the finite-dimensional l-module $S_{\mathfrak{s}_{\mathfrak{k}}}$. On this last space, there is no action of $U(\mathfrak{g})$ or $U\left(\mathfrak{k}_{\Delta}\right)$. So every eigenspace of $D(\mathfrak{g}, \mathfrak{k})^{2}$ on $V \otimes S_{\mathfrak{p}}$ just gets tensored with $S_{\mathfrak{\mathfrak { F } _ { \mathfrak { k } }}}$, and this gives the eigenspace on $V \otimes S$ for the same eigenvalue.

Since $D_{\Delta}(\mathfrak{k}, \mathfrak{l})^{2}$ commutes with $D(\mathfrak{g}, \mathfrak{k})^{2}$, it preserves these eigenspaces. Moreover, the Levi subgroup $L \subset K$ corresponding to $\mathfrak{l}$ is compact. So is then the double cover $\tilde{L}$, which acts on $V \otimes S$. Since $\tilde{L}$ commutes with $D(\mathfrak{g}, \mathfrak{k})^{2}, \tilde{L}$ also preserves the eigenspaces of $D(\mathfrak{g}, \mathfrak{k})^{2}$ and hence these eigenspaces decompose into $\tilde{L}$-irreducibles. Since $D_{\Delta}(\mathfrak{k}, \mathfrak{l})^{2}$ is up to an additive constant equal to the Casimir element of $\mathfrak{l}_{\Delta}$, it follows that $D_{\Delta}(\mathfrak{k}, \mathfrak{l})^{2}$ diagonalizes on each eigenspace of $D(\mathfrak{g}, \mathfrak{k})^{2}$. To conclude:

Lemma 7.2. $V \otimes S$ is a direct sum of eigenspaces for $\Delta$. In particular, $V \otimes S=$ $\operatorname{Ker} \Delta \oplus \operatorname{Im} \Delta$.

It is now easy to obtain a variant of the usual Hodge decomposition. The following is well known; see for instance [W], Scholium 9.4.4.

Lemma 7.3. (a) $\operatorname{Ker} \Delta=\operatorname{Ker} C \cap \operatorname{Ker} C^{\text {adj }}$

(b) $\operatorname{Im} C$ is orthogonal to $\operatorname{Ker} C^{\text {adj }}$ and $\operatorname{Im} C^{\text {adj }}$ is orthogonal to $\operatorname{Ker} C$.

Combining the two lemmas above and standard arguments, we get:

Theorem 7.4. (a) $V \otimes S=\operatorname{Ker} \Delta \oplus \operatorname{Im} C \oplus \operatorname{Im} C^{\text {adj }}$;

(b) $\operatorname{Ker} C=\operatorname{Ker} \Delta \oplus \operatorname{Im} C$;

(c) $\operatorname{Ker} C^{\text {adj }}=\operatorname{Ker} \Delta \oplus \operatorname{Im} C^{\text {adj }}$.

In other words, we have obtained a Hodge theorem for $\overline{\mathfrak{u}}$-cohomology.

To obtain it also for $\mathfrak{u}$-homology, we note that

$$
\left(C^{-}\right)^{\text {adj }}=\left(C_{\Delta}^{-}(\mathfrak{k}, \mathfrak{l})+C^{-}(\mathfrak{g}, \mathfrak{k})\right)^{\text {adj }}=C_{\Delta}(\mathfrak{k}, \mathfrak{l})-C(\mathfrak{g}, \mathfrak{k}),
$$

and so

$$
\begin{aligned}
{\left[C^{-},\left(C^{-}\right)^{\text {adj }}\right] } & =\left[C_{\Delta}^{-}(\mathfrak{k}, \mathfrak{l})+C^{-}(\mathfrak{g}, \mathfrak{k}), C_{\Delta}(\mathfrak{k}, \mathfrak{l})-C(\mathfrak{g}, \mathfrak{k})\right] \\
& =\left[C_{\Delta}^{-}(\mathfrak{k}, \mathfrak{l}), C_{\Delta}(\mathfrak{k}, \mathfrak{l})\right]-\left[C^{-}(\mathfrak{g}, \mathfrak{k}), C(\mathfrak{g}, \mathfrak{k})\right]=\Delta .
\end{aligned}
$$

So the situation for $C^{-}$is exactly the same as for $C$ and we conclude

Theorem 7.5. (a) Ker $\Delta=\operatorname{Ker} C^{-} \cap \operatorname{Ker}\left(C^{-}\right)^{\text {adj }}$;

(b) $V \otimes S=\operatorname{Ker} \Delta \oplus \operatorname{Im} C^{-} \oplus \operatorname{Im}\left(C^{-}\right)^{\text {adj }}$;

(c) $\operatorname{Ker} C^{-}=\operatorname{Ker} \Delta \oplus \operatorname{Im} C^{-}$;

(d) $\operatorname{Ker}\left(C^{-}\right)^{\text {adj }}=\operatorname{Ker} \Delta \oplus \operatorname{Im}\left(C^{-}\right)^{\text {adj }}$.

In other words, Hodge decomposition also holds for $\mathfrak{u}$-homology. Moreover, we see that $\overline{\mathfrak{u}}$-cohomology and $\mathfrak{u}$-homology have the same set of harmonic representatives, $\operatorname{Ker} \Delta$. In particular, they are isomorphic.

We now want to relate $\overline{\mathfrak{u}}$-cohomology and $\mathfrak{u}$-homology to Dirac cohomology with respect to $D(\mathfrak{g}, \mathfrak{l})$. The main observation here is

Lemma 7.6. $\operatorname{Ker} \Delta=\operatorname{Ker} D_{\Delta}(\mathfrak{k}, \mathfrak{l})^{2} \cap \operatorname{Ker} D(\mathfrak{g}, \mathfrak{k})^{2}=\operatorname{Ker} D_{\Delta}(\mathfrak{k}, \mathfrak{l}) \cap \operatorname{Ker} D(\mathfrak{g}, \mathfrak{k})$.

Proof. The operators $D_{\Delta}(\mathfrak{k}, \mathfrak{l})^{2}$ and $-D(\mathfrak{g}, \mathfrak{k})^{2}$ are both positive semidefinite and their sum is $\Delta$. This immediately implies the first equality. The second follows from $\operatorname{Ker} D_{\Delta}(\mathfrak{k}, \mathfrak{l})^{2}=\operatorname{Ker} D_{\Delta}(\mathfrak{k}, \mathfrak{l})$ (since $D_{\Delta}(\mathfrak{k}, \mathfrak{l})$ is self-adjoint) and $\operatorname{Ker} D(\mathfrak{g}, \mathfrak{k})^{2}=$ $\operatorname{Ker} D(\mathfrak{g}, \mathfrak{k})$ (since $D(\mathfrak{g}, \mathfrak{k})$ is anti-self-adjoint). 
We now relate Dirac cohomology for $D(\mathfrak{g}, \mathfrak{k})$ to $\mathfrak{p}^{+}$-homology and $\mathfrak{p}^{-}$-cohomology.

Theorem 7.7. Let $V$ be an admissible unitary $(\mathfrak{g}, K)$-module. Consider the actions of $D(\mathfrak{g}, \mathfrak{k}), C(\mathfrak{g}, \mathfrak{k}), C^{-}(\mathfrak{g}, \mathfrak{k})$ on $V \otimes S_{\mathfrak{p}}$. We have then

(a) $\operatorname{Ker} D(\mathfrak{g}, \mathfrak{k})=\operatorname{Ker} D^{2}(\mathfrak{g}, \mathfrak{k})=\operatorname{Ker} C(\mathfrak{g}, \mathfrak{k}) \cap \operatorname{Ker} C^{-}(\mathfrak{g}, \mathfrak{k})$;

(b) $V \otimes S_{\mathfrak{p}}=\operatorname{Ker} D(\mathfrak{g}, \mathfrak{k}) \oplus \operatorname{Im} C(\mathfrak{g}, \mathfrak{k}) \oplus \operatorname{Im} C^{-}(\mathfrak{g}, \mathfrak{k})$;

(c) $\operatorname{Ker} C(\mathfrak{g}, \mathfrak{k})=\operatorname{Ker} D(\mathfrak{g}, \mathfrak{k}) \oplus \operatorname{Im} C(\mathfrak{g}, \mathfrak{k})$;

(d) $\operatorname{Ker} C^{-}(\mathfrak{g}, \mathfrak{k})=\operatorname{Ker} D(\mathfrak{g}, \mathfrak{k}) \oplus \operatorname{Im} C^{-}(\mathfrak{g}, \mathfrak{k})$.

Proof. Let us simply denote by $D, C$ and $C^{-}$these operators. The first equality in (a) comes from the fact that $D$ is semi-simple, as we have already noticed. Thus, we have also $V \otimes S_{\mathfrak{p}}=\operatorname{Ker} D \oplus \operatorname{Im} D$. Since $D^{2}=C C^{-}+C^{-} C$, we can copy the proof of Lemma 7.3 and Theorem 7.4 with $\Delta$ replaced by $D^{2}$ and $C^{\text {adj }}$ replaced by $C^{-}$. This gives the results we want.

We can now combine the results above to get

Corollary 7.8. $\operatorname{Ker} \Delta=\operatorname{Ker} C_{\Delta}(\mathfrak{k}, \mathfrak{l}) \cap \operatorname{Ker} C_{\Delta}^{-}(\mathfrak{k}, \mathfrak{l}) \cap \operatorname{Ker} C(\mathfrak{g}, \mathfrak{k}) \cap \operatorname{Ker} C^{-}(\mathfrak{g}, \mathfrak{k})$.

Proof. It is obvious that the left-hand side contains the right-hand side. Conversely, if $x \in \operatorname{Ker} \Delta$, then $C x=0$ by the theorem, $D(\mathfrak{g}, \mathfrak{k}) x=0$ by the lemma, so $C(\mathfrak{g}, \mathfrak{k}) x=0$ by Theorem 7.7 and so also $C x-C(\mathfrak{g}, \mathfrak{k}) x=C_{\Delta}(\mathfrak{k}, \mathfrak{l}) x=0$. Analogously, $C^{-}(\mathfrak{g}, \mathfrak{k}) x=0$ and $C_{\Delta}^{-}(\mathfrak{k}, \mathfrak{l}) x=0$.

Since $\operatorname{Ker} C_{\Delta}(\mathfrak{k}, \mathfrak{l}) \cap \operatorname{Ker} C(\mathfrak{g}, \mathfrak{k})$ can be thought of as the kernel of $C_{\Delta}(\mathfrak{k}, \mathfrak{l})$ acting on the kernel of $C(\mathfrak{g}, \mathfrak{k})$, and similarly for the $C^{-}$-operators, we can reinterprete the corollary as follows:

Corollary 7.9. To calculate the $\overline{\mathfrak{u}}$-cohomology of $V$, one can first calculate the $\mathfrak{p}^{-}$-cohomology of $V$ to obtain a $\tilde{K}$-module, and then calculate the $\overline{\mathfrak{u}} \cap \mathfrak{k}$-cohomology of this module. Analogously, to calculate the $\mathfrak{u}$-homology of $V$, one can first calculate the $\mathfrak{p}^{+}$-homology of $V$, and then the $\mathfrak{u} \cap \mathfrak{k}$-homology of the resulting $\tilde{K}$-module.

Remark 7.10. Note that this is, in fact, the Hochschild-Serre spectral sequence for the ideal $\mathfrak{p}^{-}$of $\overline{\mathfrak{u}}$, respectively, the ideal $\mathfrak{p}^{+}$of $\mathfrak{u}$. What we have obtained is that these Hochschild-Serre spectral sequences are always degenerate for a unitary $(\mathfrak{g}, K)$-module $V$.

We now turn our attention to the Dirac cohomology of $D=D(\mathfrak{g}, \mathfrak{l})$. In addition to the above considerations, we bring in Corollary [5.2, and note that for both $D_{\Delta}(\mathfrak{k}, \mathfrak{l})$ and $D(\mathfrak{g}, \mathfrak{k})$ the cohomology is the same as the kernel or the kernel of the square. Thus, we obtain:

Theorem 7.11. The Dirac cohomology $H_{D}(\mathfrak{g}, \mathfrak{l} ; V)$ of a unitary $(\mathfrak{g}, K)$-module $V$ is isomorphic to the $\overline{\mathfrak{u}}$-cohomology of $V$ and the $\mathfrak{u}$-homology of $V$ up to appropriate modular twists. Moreover, all three cohomologies have the same set of harmonic representatives, $\operatorname{Ker} \Delta$.

\section{REFERENCES}

[AM] A. Alekseev, E. Meinrenken, Lie theory and the Chern-Weil homomorphism, Ann. Sci. École Norm. Sup. (4) 38 (2005), no. 2, 303-338. MR2144989 (2006d:53020)

[BW] A. Borel, N. Wallach, Continuous cohomology, discrete subgroups, and representations of reductive groups, Second edition. Mathematical Surveys and Monographs, 67, American Mathematical Society, Providence, RI, 2000. MR1721403 (2000j:22015) 
[HP1] J.-S. Huang, P. Pandžić, Dirac cohomology, unitary representations and a proof of a conjecture of Vogan, J. Amer. Math. Soc. 15 (2002), 185-202. MR.1862801 (2002h:22022)

[HP2] J.-S. Huang, P. Pandžić, Dirac operators in representation theory, Representations of Real and $p$-adic Groups, Lecture Notes Series, Vol. 2, Institute for Mathematical Sciences, National University of Singapore, Singapore University Press and World Scientific, 2004, pp. 163-219. MR2090871 (2005f:22022)

[K1] B. Kostant, Lie algebra cohomology and the generalized Borel-Weil theorem, Ann. of Math. 74 (1961), 329-387. MR0142696 (26:265)

[K2] B. Kostant, A cubic Dirac operator and the emergence of Euler number multiplets of representations for equal rank subgroups, Duke Math. J. 100 (1999), 447-501. MR:1719734 (2001k:22032)

[K3] B. Kostant, A generalization of the Bott-Borel-Weil theorem and Euler number multiplets of representations, Lett. Math. Phys. 52 (2000), 61-78. MR.1800491 (2001m:22028)

[K4] B. Kostant, Dirac cohomology for the cubic Dirac operator, Studies in Memory of I. Schur, Progr. Math. vol. 210 (2003), 69-93. MR1985723 (2004h:17005)

[Ku] S. Kumar, Induction functor in non-commutative equivariant cohomology and Dirac cohomology, J. Algebra 291 (2005), no. 1, 187-207. MR2158518 (2006e:17029)

[P] R. Parthasarathy, Dirac operator and the discrete series, Ann. of Math. 96 (1972), 1-30. MR0318398 (47:6945)

[V1] D.A. Vogan, Representations of real reductive Lie groups, Birkhäuser, Boston, 1981. MR0632407 (83c:22022)

[V2] D.A. Vogan, Dirac operators and unitary representations, 3 talks at MIT Lie groups seminar, Fall 1997.

[V3] D.A. Vogan, n-cohomology in representation theory, a talk at "Functional Analysis VII", Dubrovnik, Croatia, September 2001.

[W] N.R. Wallach, Real Reductive Groups, Volume I, Academic Press, 1988. MR0929683 (89i:22029)

Department of Mathematics, Hong Kong University of Science and Technology, Clear Water Bay, Kowloon, Hong Kong SAR, China

E-mail address: mahuang@ust.hk

Department of Mathematics, University of Zagreb, BijeničKa 30, 10000 Zagreb, Croatia

E-mail address: pandzic@math.hr

Centre de Mathématiques Laurent Schwartz, Ecole Polytechnique, 91128 Palaiseau Cedex, France

E-mail address: renard@math.polytechnique.fr 